\title{
¿Cómo calcular una estructura? Nuevos planteamientos docentes
}

\author{
ESPERANZA RODRÍGUEZ MAYORGA \\ Universidad de Sevilla \\ Departamento de Estructuras de \\ Edificación e Ingeniería del Terreno \\ espe@us.es \\ D.0.I.: http://dx.doi.org/10.12795/JDU.2018.i01.36 \\ Pp.: 637-654
}

\section{Resumen}

La asignatura de Estructuras 1 es una de las más complejas de cuantas tendrán que afrontar los alumnos de primer curso del grado en Fundamentos de Arquitectura. En ella se dan los primeros pasos dentro del cálculo de estructuras, sentando las bases de esta disciplina. Es importante que adquieran esta competencia fundamental pues la usarán reiteradamente en los siguientes años de carrera. El ciclo de mejora que se recoge en este documento abarca fundamentalmente dos aspectos: la exploración de nuevas formas de docencia y la ligera ampliación o modificación del contenido de la asignatura establecido. El objeto de tal ampliación ha sido ajustar el proceso de cálculo de estructuras que se sigue en clase al proceso habitual que se sigue en la práctica profesional o que siguen los programas informáticos de cálculo (manejados además por los alumnos dentro de la misma asignatura).

Palabras clave: Estructuras 1, Grado en Fundamentos de Arquitectura, Docencia Universitaria, Experimentación docente universitaria, Fundamentos del Diseño y Cálculo de Estructuras de Edificación.

Esta obra se distribuye con la licencia Creative Commons Reconocimiento-NoComercial-SinObraDerivada $\quad 4.0$ Internacional (CC BY-NC-ND 4.0.) 


\section{Contexto}

La asignatura Estructuras 1 se enmarca en un conjunto de tres asignaturas obligatorias impartidas en el Grado en Fundamentos de Arquitectura (Plan 2012, Escuela Técnica Superior de Arquitectura de Sevilla): Estructuras 1 (segundo semestre del primer curso), Estructuras 2 (primer semestre del tercer Curso) y Estructuras 3 (segundo semestre del cuarto curso). En estrecha relación se halla la asignatura de Física 1, impartida en el primer semestre del primer curso, que abarca temas procedimentales necesarios para la docencia de estructuras. Mientras que en Estructuras 1 se tratan los fundamentos del diseño y del cálculo estructural comunes a todos los sistemas (Torroja, 1957), Estructuras 2 se centra en estructuras de acero y Estructuras 3 en estructuras de hormigón.

Las aulas donde se imparte la docencia cuentan con mobiliario formado por mesas y sillas no fijas, lo que permite realizar cambios en la disposición del mobiliario según necesidades. Los estudiantes cuentan con abundante material para el desarrollo de la asignatura: bibliografía de carácter general, bibliografía específica y un temario adaptado a sus posibilidades de comprensión. Además, como parte de esta mejora de la asignatura, se les ha proporcionado la normativa vigente y formularios comerciales de perfiles de acero normalizados. El número de alumnos por grupo es reducido, entre 25 y 30 alumnos, lo que da lugar a seis equipos de trabajo en cada uno de los grupos.

\section{Diseño del Ciclo de Mejora Docente}

El objetivo del ciclo de Mejora Docente era acercar al alumno a la problemática real que se le planteará a lo largo de su vida profesional, que es calcular una estructura, además de ser capaz de interpretar la normativa al respecto y aplicarla a dicho proceso. El diseño de la asignatura en su conjunto responde a acercar al alumno a un problema real, profesional, siguiendo las directrices documentadas en la bibliografía (Bain, 2007; Finkel, 2008; Giné 
Freixes, 2009; Meier, 2000). Concretamente, el ciclo de mejora se centra en las comprobaciones normativas (Código Técnico, 2006) a realizar a una estructura ya sean relativas a evitar el colapso de la estructura (Estados Límite Últimos, ELU) o a evitar situaciones que impidan el normal uso de la edificación (Estados Límite de Servicio, ELS).

\section{Mapa de contenidos}

El mapa de contenidos para este ciclo de mejora es, de hecho, el de la asignatura completa, pues la asignatura de Estructuras 1 presenta un esquema repetitivo en torno al proceso de cálculo de estructuras: diseño, cálculo y comprobación de una estructura isostática de una sola barra (vigueta); diseño, cálculo y comprobación de una estructura plana hiperestática de varias barras (vigueta continua y pórtico plano); diseño, cálculo y comprobación de una estructura espacial hiperestática de varias barras (edificio). Este proceso repetitivo va precedido de unas lecciones donde se trata el análisis de esfuerzos, herramienta indispensable y fundamental. El ciclo de mejora que aquí se describe se centra en las dos unidades centrales (diseño, cálculo y comprobación de viguetas isostáticas e hiperestáticas y pórticos).

El mapa de contenidos se ha ido presentando a los alumnos poco a poco, es decir, se ha ido construyendo dentro de la propia clase. Inicialmente se planteó como algo muy simplificado, la sucesión de tres planteamientos: diseño, acciones o cargas y comprobaciones de ELU y ELS (Figura 1).

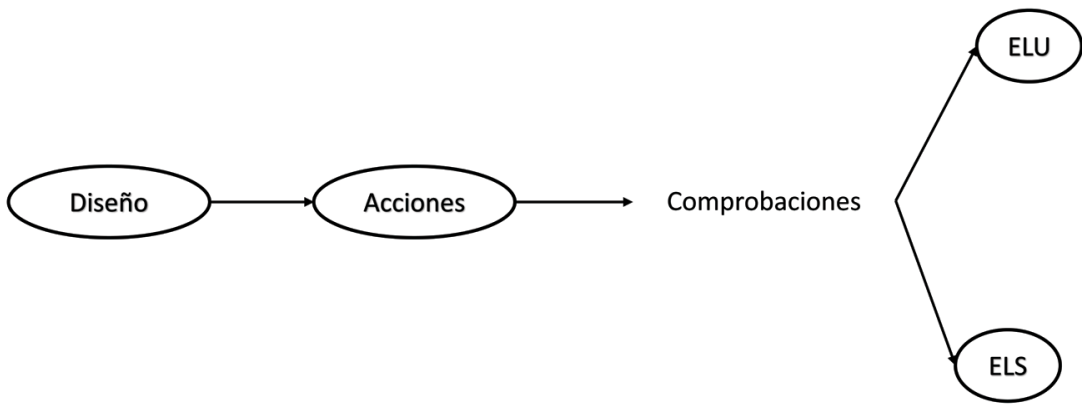

Figura 1. Mapa conceptual inicial de la asignatura Estructuras 1

Jornadas de Formación e Innovación Docente del Profesorado | № 1 (2018) Esta obra se distribuye con la licencia Creative Commons 
Los alumnos asimilaron esta sucesión perfectamente, pues es del todo lógica. Una vez los alumnos ya habían diseñado una estructura para un edificio real (edificios sencillos que presentan un nivel de dificultad muy bajo para diseñarles una estructura, escogidos antes de la primera semana de clase y asignados a cada grupo de alumnos) y le habían introducido las cargas definiendo sus hipótesis simples, se planteó la necesidad del primero de los conceptos fundamentales sobre el que versa la asignatura: los esfuerzos.

De este modo, los esfuerzos surgieron como una necesidad, la herramienta necesaria para poder realizar las comprobaciones. La siguiente ampliación del mapa se produjo una vez los alumnos habían comprendido las dos formas posibles de colapso de las estructuras. De esta forma, el mapa conceptual se fue completando hasta llegar a su versión final (Figura 2).
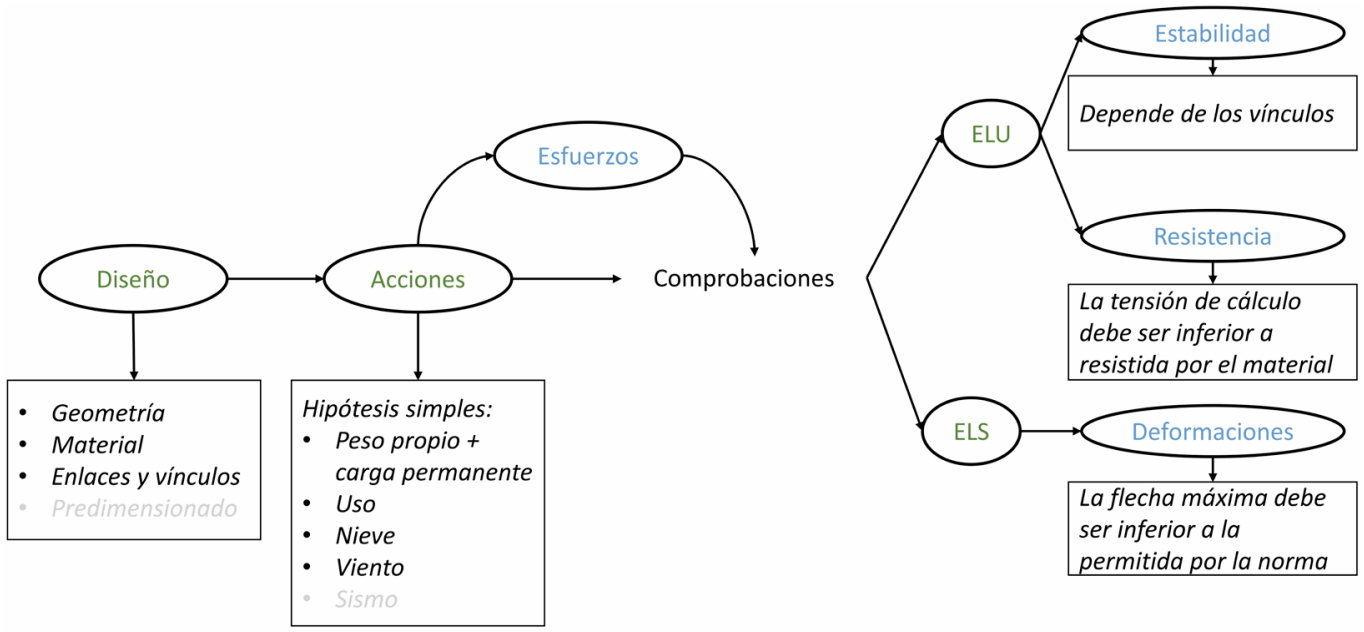

Figura 2. Mapa conceptual completo de la asignatura Estructuras 1.

Jornadas de Formación e Innovación Docente del Profesorado | № 1 (2018)

Esta obra se distribuye con la licencia Creative Commons

Reconocimiento-NoComercial-SinObraDerivada

Internacional (CC BY-NC-ND 4.0.) 


\section{Modelo metodológico}

La metodología docente seguida en la asignatura es el Aprendizaje Basado en Problemas (ABP) (De Justo, 2013). El desarrollo habitual de una clase previa al ciclo de mejora objeto de esta comunicación seguiría el esquema de la figura 3 (De Alba y Porlán, 2017).

\section{TEORÍA $\rightarrow$ PROBLEMA $\rightarrow$ CONCLUSIONES}

Figura 3. Modelo metodológico inicial de la asignatura Estructuras 1.

Esto, de forma un poco más detallada, podría resumirse en que los alumnos antes del comienzo de la clase se han estudiado la teoría correspondiente al tema que se tratará. Además, han elaborado resúmenes que entregan y con los cuales el alumno podrá contar durante la realización de los controles de la asignatura. En clase se aclaran las dudas que hayan podido surgir del estudio individual y se plantea un problema para su aplicación.

La realidad observada es que el alumno no es capaz de aplicar la teoría a la práctica, sino que es al contrario, inducen la teoría en lugar de deducir la práctica. Es por ello que el modelo metodológico al que se pretende llegar es aquel en el que una teoría ordenada y sistemática sea el fruto de las conclusiones obtenidas de la práctica y del debate entre los alumnos. Es decir, partiendo de breves nociones teóricas (las mínimas necesarias), los alumnos se enfrentan a un problema, para cuya resolución les faltan herramientas teóricas que deben buscar. A partir de la puesta en común dentro y fuera de su equipo de trabajo, obtienen unas conclusiones que constituyen la teoría (Figura 4).

$$
\text { TEORIA } \rightarrow \text { PROBLEMA } \rightarrow \text { PUESTA EN COMÚN } \rightarrow \text { CONCL. }=\text { TEORÍA }
$$

Figura 4. Modelo metodológico ideal de la asignatura Estructuras 1.

Este modelo presenta una problemática importante, que es la limitación temporal de que se dispone. Es por 
ello que determinadas actividades, como la puesta en común a nivel de toda la clase, se realiza en horario no presencial, sin ningún control por parte del profesor y sin ninguna seguridad de que se ha llevado a cabo correctamente. Este aspecto es claramente a mejorar.

\section{Cuestionario inicial-final}

Antes y después del ciclo de mejora que aquí se describe se pasó un cuestionario a rellenar por los estudiantes. Se invitó a los estudiantes a completarlo de manera individual y a identificarse en los mismos. Se plantearon tres cuestiones:

1. Las cargas teóricas sabemos que son de tres tipos: puntuales, lineales, y superficiales. Indica a que tipo teórico corresponde cada una de las siguientes acciones sobre la estructura (es decir, en 3D):

a) La nieve:

b) La sobrecarga de uso:

c) Un cerramiento:

d) La tabiquería:

e) Un cartel publicitario soportado por dos perfiles y apoyado sobre la cubierta del edificio.

2. Dentro del proceso habitual de cálculo de una estructura, las combinaciones son situaciones de dimensionado posibles que ocurran. ¿En qué momento del dimensionado deben aplicarse? ¿En qué unidades se obtiene su resultado?

3. El paso "clave" dentro del dimensionado de la estructura es el paso de "esfuerzo" a "tensión". Este paso implica un cambio en las unidades entre ambos conceptos. Indica, de manera aproximada, cómo se realiza este paso para cada uno de los esfuerzos que estamos tratando en el Módulo 2. 
Las tres preguntas estaban planteadas para dar respuesta a varias dudas. Por un lado, es necesario conocer el nivel del alumnado en términos de conocimiento, es decir, qué conceptos manejan y cuáles no, en resumen, qué saben. Por otro lado, hay otras características actitudinales y aptitudinales que es preciso conocer por parte del profesor (capacidad de abstracción, lógica matemática y comprensión de conceptos complejos).

Los cuestionarios se pasaron antes y después de la realización de unas actividades en las que se trabajarían los contenidos conceptuales y actitudes y aptitudes que en el cuestionario se exploran. También se volvieron a pasar a final del curso. Los resultados extraídos se presentarán y analizarán en el apartado correspondiente.

\section{Aplicación del Ciclo de Mejora Docente}

\section{Relato resumido de las sesiones}

En las sesiones correspondientes al ciclo de mejora se han realizado cuatro actividades. La duración aproximada de cada una de ellas ha sido de 2 horas en cada uno de los dos grupos en los que se ha realizado esta experiencia. Por tanto, el ciclo, de 8 horas, se experimentó dos veces. Presento a continuación las actividades desarrolladas.

Actividad 1: Interpretación de la normativa vigente y aplicación a una vigueta isostática.

La asignatura de Estructuras 1 versa sobre el análisis y dimensionado genérico de estructuras. Este análisis se realiza según el método de los Estados Límite recogido en el Código Técnico de la Edificación, normativa de obligado cumplimiento en España (Código Técnico, 2006).

Sentido educativo de la actividad: Esta actividad tiene como objetivo que los alumnos se enfrenten por primera vez a una normativa real. Desde el punto de vista didáctico, 
trabajar con normativa implica una dificultad añadida a la de trabajar con textos adaptados. Esta actividad, además, les ayuda a controlar la nomenclatura específica en la que se están iniciando en este momento.

Descripción detallada: La actividad se desarrolló en el aula en los mismos grupos de trabajo en los que los alumnos llevan trabajando durante todo el curso. Se siguieron los siguientes pasos dentro de la actividad:

- Reparto en papel de las páginas de la normativa española sobre el método de los estados límite, concretamente del "Documento Básico Seguridad Estructural" (Código Técnico, 2006) y sobre el apartado donde se describen las "Verificaciones basadas en coeficientes parciales", comúnmente conocidas como las "Combinaciones".

- Vagas aclaraciones por parte del profesor sobre la misma.

- Lectura comprensiva de los alumnos del texto proporcionado. Se les insiste en que están haciendo algo real, lo que a algunos les sirve de motivación y a otros de lo contrario. Ninguno le encuentra correlación con el texto simplificado y adaptado que forma parte de la información que se les suministró a principios de curso, y que ya han estudiado y resumido en una sesión anterior.

- Se comienza un debate a nivel de la clase, donde cada grupo de alumnos expone lo que ha entendido del texto que han leído. Entre todos, y con la orientación del profesor, se va llegando a la conclusión de cómo interpretar la norma, de complejidad elevada para ellos.

- Se aplican las combinaciones a la práctica de curso en la que están trabajando en ese momento (vigueta biapoyada sometida exclusivamente a cargas verticales).

- Exposición en la pizarra de cómo se aplicaría el método a una vigueta del tipo analizado. 
- Finalización de la actividad en horario no presencial, pues ningún grupo tiempo suficiente en las dos horas establecidas para concluir la actividad.

Contenidos manejados: Los contenidos manejados en esta actividad se centran en la última parte del mapa conceptual de la asignatura (Figura 5). Se han trabajado las comprobaciones de Estados Límite Últimos y de Servicio (ELU y ELS) extrayéndolas de la normativa correspondiente.

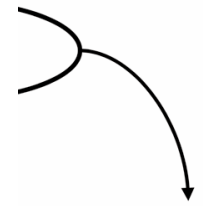

Comprobaciones

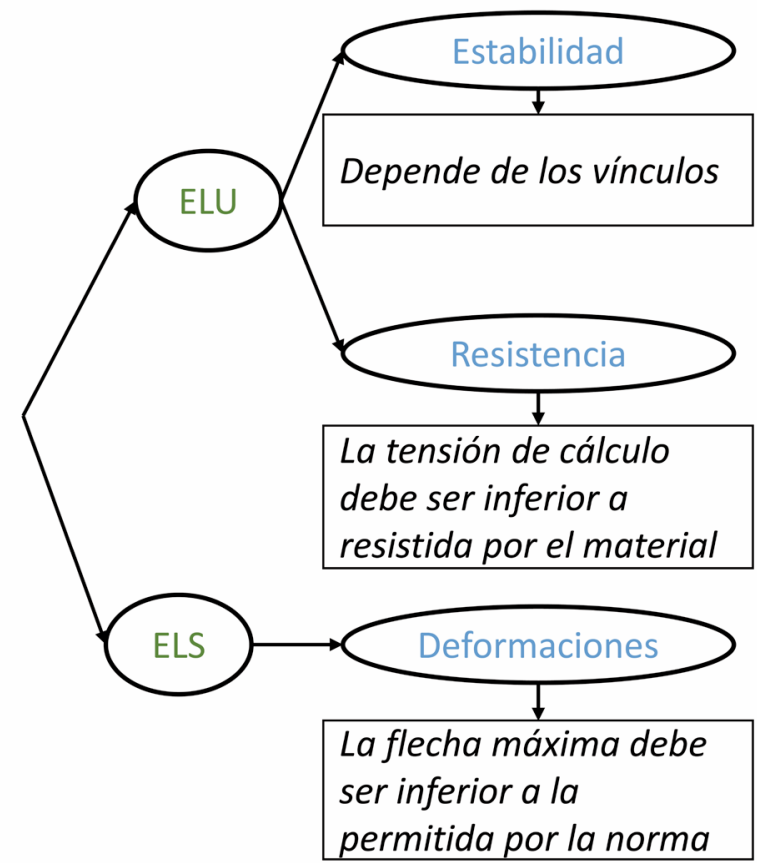

Figura 5. Contenidos trabajados en la primera actividad del segundo ciclo de mejora.

Jornadas de Formación e Innovación Docente del Profesorado | № 1 (2018)

(ic) (i) Esta obra se distribuye con la licencia Creative Commons Reconocimiento-NoComercial-SinObraDerivada Internacional (CC BY-NC-ND 4.0.) 
Actividad 2: Análisis de una vigueta isostática mediante un programa de cálculo informático y comparación con el resultado manual.

El cálculo manual es una disciplina que es preciso controlar. Es indispensable "saber hacer" para "saber ordenar qué hacer". Por otro lado, la potencia de cálculo de los ordenadores y programas de cálculo, así como la accesibilidad de los mismos para el alumnado es algo que hay que aprovechar. En este contexto se enmarca la segunda actividad.

Sentido educativo de la actividad: En esta actividad se introduce a los alumnos en el manejo de un programa de cálculo comercial, el conocido a nivel profesional CYPE 3D (CYPE, 2108). La Universidad de Sevilla cuenta con licencia para su uso. El objetivo no es aprender a manejar el programa, sino aprender a interpretar y validar los resultados extraídos del programa.

Descripción detallada: La actividad consiste en calcular la misma vigueta que dimensionaron en la actividad 1 usando ahora otro método, y comparar los resultados obtenidos con el cálculo manual y el informático. En esta comparativa, el cálculo manual juega el papel de corrección del informático y viceversa. Se siguieron los siguientes pasos:

- El profesor estableció en la pizarra el problema que se va a resolver usando CYPE. El problema era similar al de los alumnos, pero no coincidía con ninguno de ellos. El profesor trabajó en su ordenador proyectando su análisis.

- Se introducen los datos generales de la obra: (materiales, localización, nivel de control...) y los datos específicos. El profesor los que ha planteado en la pizarra y cada grupo los suyos propios.

- Se calcula la estructura y se obtienen los resultados. Se comprueba que los resultados obtenidos se encuentran dentro de lo razonable: la deformada es coherente, los signos de los esfuerzos también, etc. 
- Se comparan los resultados obtenidos en el ordenador con los calculados por los alumnos manualmente al final de la actividad 1. De esta comparación se obtuvieron conclusiones muy importantes.

Contenidos manejados: En esta actividad se recorre el mapa conceptual prácticamente completo de la asignatura (figura 2), ya que se calcula una estructura sencilla completa usando un programa informático. Quedaría excluido de esta actividad solo el diseño de la estructura.

Actividad 3: Diseño y análisis de una vigueta hiperestática.

Esta actividad supone dar el salto entre las estructuras isostáticas y las hiperestáticas (la mayoría de estructuras de edificación). En este caso, el cálculo informático ya se hace imprescindible. También se introducen en el "número gordo" (Arroyo Portero, 2009) como comprobación del cálculo informático.

Sentido educativo de la actividad: Los objetivos de esta actividad son varios:

Analizar pros y contras de los sistemas hiperestáticos frente a los isostáticos.

Tomar conciencia de la importancia del cálculo manual como comprobación del cálculo informático y para tener una idea del resultado incluso antes de comenzar a calcular.

Continuar practicando la introducción de datos en CYPE 3D (CYPE, 2018).

Descripción detallada: La actividad es similar a la actividad 2. La principal diferencia estriba en el modelo analizado, hiperestático en este caso. Los grupos de trabajo van funcionando de manera independiente con la supervisión del profesor. Los pasos seguidos fueron:

- Los alumnos resuelven el forjado de su proyecto ahora usando viguetas hiperestáticas, es decir, viguetas continuas. 
- Introducen los datos en CYPE 3D (CYPE, 2018). Concluyen que las cargas no se modifican respecto a la vigueta isostática.

- Calculan, obtienen resultados, y los validan, mediante su comparación con los datos calculados manualmente para la vigueta isostática.

- Comparan los resultados de ambos sistemas, isostático e hiperestático, para la solución del mismo problema. Las ventajas del sistema hiperestático, prácticamente todos la reducen al ahorro económico en términos de kilos de acero empleados en la obra. No son capaces de reflexionar sobre la mayor seguridad que ofrecen las estructuras hiperestáticas frente a las isostáticas.

Contenidos manejados: Esta actividad abarca la totalidad del mapa conceptual de la asignatura (Figura 2). Los alumnos comienzan diseñando la vigueta, aplican cargas, obtienen esfuerzos y realizan comprobaciones. Parte de los contenidos son, por tanto, reiterativos.

Actividad 4: Análisis de los diagramas de esfuerzos de un pórtico plano.

El sistema estructural principal más sencillo es el pórtico plano. En esta actividad, los alumnos deben deducir los ítems que marcan el comportamiento de un pórtico plano de nudos rígidos de dos soportes y una altura, en términos de esfuerzos y deformaciones. Su organización se basa en los "Talleres conceptuales" (Finkel, 2008), aunque no coincida exactamente con la definición.

Sentido educativo de la actividad: La finalidad de los alumnos es comenzar a comprender el funcionamiento de las estructuras hiperestáticas de varias barras: el pórtico. Mediante la manipulación de las propiedades tangibles de sección y dimensiones geométricas del pórtico, los alumnos aprendieron los conceptos de reparto de esfuerzos en el nudo y rigidez a flexión. 
Descripción detallada: La actividad transcurrió durante dos horas presenciales. Consistió en partir de un problema propuesto por el profesor para el que los alumnos no pueden dar respuesta por falta de herramientas en ese momento. Es más dejándose llevar por la intuición y por la aplicación de los conocimientos que han adquirido hasta el momento, darán una respuesta errónea. A partir de este momento, se les dan pistas sucesivas para que los alumnos descubran, por un lado, la incoherencia de la solución inicial propuesta, y por otro, la solución correcta al problema. La secuencia de actividades seguida consistió en:

Pregunta à respuesta de un alumno voluntario en la pizarra à Opiniones de los demás à Respuesta de otro alumno... y así sucesivamente hasta que ninguna persona encontrase ningún inconveniente a la respuesta aportada:

- Planteamiento de un problema: se dibuja en la pizarra un pórtico de dos pilares y una altura, del que no se da más información que el trazado de los ejes de las barras que lo componen. Este pórtico está solicitado por una carga vertical continua sobre la viga. Los nudos son rígidos y los pilares están empotrados en su sección inferior. Se les pide a los alumnos dibujar la deformada. Pregunta "trampa" de partida a la que dan una respuesta errónea que ellos mismos deducirán su incorrección al final de la actividad.

- Pista 1: secciones de las barras. Primero se les da un perfil de viga elevado en la serie y muy bajo para los pilares; segundo, perfil de viga muy bajo en la serie y elevado para los pilares. En estos dos supuestos, dibujan la deformada.

- Pista 2: dibujan deformadas dos vigas, una biapoyada y otra biempotrada. Se pide que asimilen cada una con la viga los pórticos de la pista 1 en términos de deformaciones.

- Pista 3: Deducción de los signos de los flectores a partir de las deformadas. 
- Pista 4: Asimilación de cada pórtico a las vigas de la pista 2 en términos de esfuerzos.

- Pista 5: Dibujan las leyes de esfuerzos en el pórtico completo de forma que sean coherentes con todo lo que se ha ido viendo en la pizarra.

Finalmente se llega a los conceptos de equilibrio en el nudo, reparto de esfuerzos en el nudo, y se enlaza finalmente con la utilidad del predimensionado para el cálculo informático.

Es de destacar que la función del profesor en esta actividad fue la de ir dando las pistas sucesivas necesarias para llegar a la solución, pero que no indicó la corrección o incorrección de los resultados planteados por los alumnos. Los alumnos se implicaron en la realización de esta actividad y prácticamente todos participaron. El debate generado en clase fue muy interesante, y también sirvió para redireccionar las clases sucesivas.

Contenidos manejados: Los contenidos del mapa conceptual con los que se trabajó en esta actividad se limitaron a esfuerzos y deformaciones (Figura 2).

\section{Evaluación del aprendizaje de los estudiantes}

La evaluación del aprendizaje de los estudiantes se ha realizado mediante la propia observación y mediante el cuestionario diseñado para tal efecto.

Los resultados del cuestionario pasado a los alumnos antes y después del ciclo de mejora dieron algunos resultados esperados y otros no. Complementariamente y de forma externa al ciclo de mejora, se volvieron a pasar los cuestionarios al final del curso. Los resultados del cuestionario se han completado con las notas del "Diario del profesor", donde se van apuntando las cuestiones reseñables ocurridas y observadas en clase. En total se encuestaron 
aproximadamente 40 alumnos pertenecientes a los dos grupos en los que se ha llevado a cabo la experiencia aquí descrita. A nivel particular, para cada uno de los ítems analizados mediante los cuestionarios, se han graduado las respuestas de los estudiantes utilizando la técnica de la "escalera de aprendizaje" (Rivero y Porlán, 2017).

Las tres escaleras desarrolladas (Figura 6) demuestran el avance que han experimentado los alumnos. En general, los resultados muestran mejora de los alumnos, pero no todo lo que se esperaría. Incluso podría afirmarse que la mejora alcanzada en el ciclo ha sido la obtenida durante el curso, ya que los resultados de los cuestionarios obtenidos en fin de curso han sido análogos a los obtenidos al final del ciclo de mejora. Los peores resultados han sido los obtenidos en términos de "Comprensión", cuestión que es justificable ya que el ítem analizado es elevadamente complejo. Independientemente, los resultados obtenidos han sido mucho mejores de lo esperado. A fin de curso, los resultados no siguieron mejorando como se esperaba. Incluso podría decirse a la vista de los cuestionarios que algunos resultados han sido peores (esto puede tener relación con el hecho de que la muestra de estudiantes no ha sido siempre la misma, ya que no siempre todos los alumnos han asistido a clase).

Jornadas de Formación e Innovación Docente del Profesorado | № 1 (2018) Esta obra se distribuye con la licencia Creative Commons 

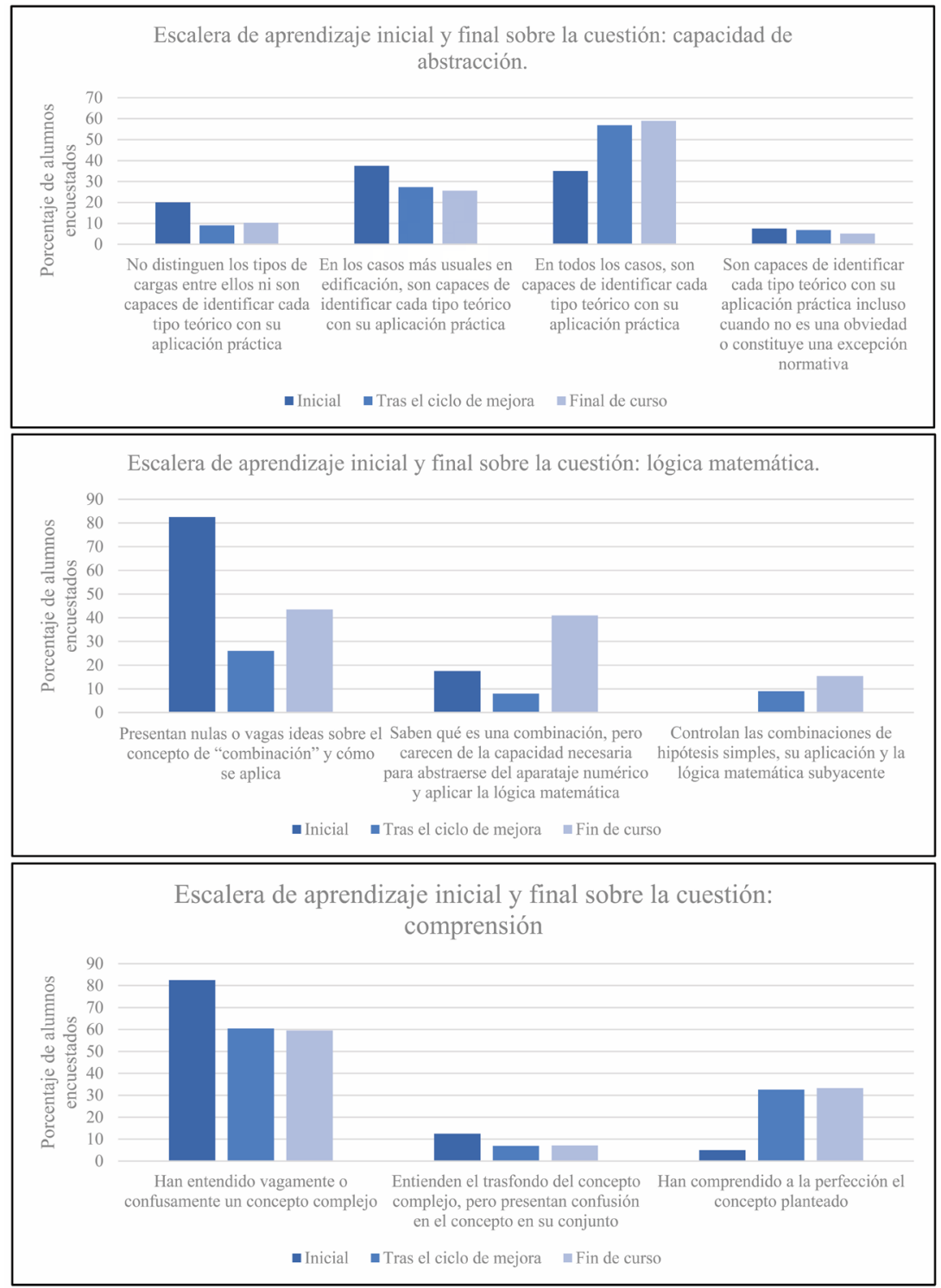

Figura 6. Escaleras de aprendizaje sobre la capacidad de abstracción (arriba), lógica matemática (centro) y comprensión de conceptos complejos (abajo). 


\section{Conclusiones: Evaluación del ciclo de mejora docente}

En líneas generales, el resultado de la aplicación del ciclo aquí descrito ha sido bastante satisfactorio. Los aspectos que han supuesto una clara mejora en la asignatura (el uso de la normativa vigente como material docente, el uso del diario del profesor como evaluación de los alumnos y de las clases, y la actividad 4, desarrollada para este ciclo de mejora, que ha producido resultados extraordinarios) se mantendrán para los cursos venideros. Igualmente, se seguirá fomentando capacidad de lectura, comprensión y lógica necesarias para el desarrollo de cualquier trabajo profesional. Por último, es importante reseñar que los tipos de metodologías "activas", implican un esfuerzo por parte del profesor y del alumno. En ocasiones, esta inversión resulta poco gratificante.

Jornadas de Formación e Innovación Docente del Profesorado | № 1 (2018) Esta obra se distribuye con la licencia Creative Commons 


\section{Referencias bibliográficas}

Arroyo Portero, J. C et al. (2009). Números gordos en el proyecto de estructuras. Edición corregida y ampliada. España: Cinter Divulgación Técnica.

Bain, K. (2007). Lo que hacen los mejores profesores de universidad. Valencia: Universitat de Valencia, Servicio de Publicaciones.

De Alba, N. y Porlán, R. (2017). La metodología de la enseñanza. En R. Porlán (Coord.), Enseñanza universitaria. Como mejorarla (pp. 37-53). Madrid: Morata.

Dirección General de Arquitectura, Vivienda y Suelo, Ministerio de Fomento (2006). Código Técnico de la Edificación. Madrid: Instituto de Ciencias de la Construcción Eduardo Torroja, CSIC. https://www.codigotecnico.org/

(c) CYPE Ingenieros, S.A. (2018). http://cype3d.cype.es/

Finkel, D. (2008). Dar clase con la boca cerrada. Valencia: Universitat de Valencia, Servicio de Publicaciones.

Giné Freixes, N. (2009). Como mejorar la docencia universitaria: el punto de vista del estudiantado. Revista Complutense de Educación, 20(1), 117-134.

De Justo Moscardó, E. (2013). Diseño y Evaluación de un Programa para el Aprendizaje de Estructuras de Edificación Mediante ABP (Tesis Doctoral). Universidad de Sevilla, Sevilla.

Meier, M. (2000). La caja negra. Journal of Materials Education, 22(4-6), 167-170.

Rivero A. y Porlán, R. (2017). La evaluación de la enseñanza universitaria. En R. Porlán (Coord.), Enseñanza universitaria. Como mejorarla (pp. 37-53). Madrid: Morata.

Torroja, E. (1957). Razón y ser de los tipos estructurales. Madrid: Consejo Superior de Investigaciones Científicas.

Jornadas de Formación e Innovación Docente del Profesorado | № 1 (2018) Esta obra se distribuye con la licencia Creative Commons 